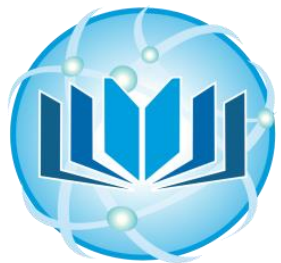

\title{
Is Chronic Shame a Life Sentence?
}

\section{Rivka A Edery}

Affiliation: School of Behavioral Sciences, Southern California University, USA

*Corresponding author: Rivka A Edery, M.S.W, L.C.S.W, School of Behavioral Sciences, Southern California University, USA, Tel: (361)

704-4051, E-mail: rebecca.edery@ gmail.com

Citation: Edery RA. Is chronic shame a life sentence? (2019) Edelweiss Psyi Open Access 3: 22-24.

Received: Jul 02, 2019

Accepted: Jul 10, 2019

Published: Jul 17, 2019

Copyright: ( ) 2019 Edery RA. This is an open-access article distributed under the terms of the Creative Commons Attribution License, which permits unrestricted use, distribution, and reproduction in any medium, provided the original author and source are credited.

\begin{abstract} conflict resolution.

Keywords: Shame, Projection, Approval, Guilt, Self-honesty.

Abbreviations: PTSD- Post Traumatic Stress Disorder.

\section{Background}

Based on the extensive research and data available, psychologists consider that shame cultivates from the need for approval from others. Shame can result when a person senses, or experiences, that someone disapproves of them or something about them. Shame also can result when a person actually did something shameful, like doing harm or damage to a person, their property, or to animals. A person can also experience shame as an adult, when something happens that they have little or no control over.
\end{abstract}

Shame is a painful, universal emotional response to a failure, shortcoming, or other wrongdoing of what society demands or what a person demands of him or herself. Scientists have learned that very young infants experience shame (as a personal feeling of unworthiness and shame), when they experience rejection-even if it is unintentional. Children, who are admonished for doing something over which they have limited or no control, are also capable of feeling shame. On a more serious, long-lasting scale, physical and sexual abuse and other traumatic experiences, particularly those that happen directly to the child, without adequate intervention, may lead to the development of shame as well. Considering the origin of the shame-wound is critical as well. If one is the child of abusive and/or addicted parents, their shame could have been an emotional and psychological 'transplant'. Assessing if the shame is a consequence of actions, inactions, or someone else's conflicts, can allow for internal

For example, being unable to afford an expensive vehicle, take a vacation, or other items that peers or neighbors may be sporting. Another common example is shame with spilling drinks or food, especially in public places, or with family members that have a history of shaming members over [1]. It is important to distinguish between shame and guilt, two emotions that are not the same, although they can be experienced at the same time, from the same cause. Guilt is an emotional reaction to a specific behavior, or failure to act on something. The theme of guilt is having done something wrong, and that one must make an amends.

Since guilt is usually attached only to that certain failing, it dispels once the person makes a proper amends. In contrast to guilt, shame is a pervasive feeling of not measuring up, or of downright failure. Sometimes the feeling is fleeting and is a result of a small breach of social norms. For example, mindlessly spitting gum on to the street instead of properly putting it in a garbage can. In more serious and prolonged sense of unworthiness, shame can be the consequence of cold disapproval, toxic rejection, abuse or neglectof the person's parent, caregiver, or significant other [1].

\section{The Role of Cognition in Shame}

The distress caused by shame can be inspired by both positive and a negative cognition and motivation. The thinking that supports socially acceptable behaviors, like dressing appropriately, keeping one's work area neat and clean, and not littering, are examples of positive cognition utilized to avoid shame. The motivation is a positive and necessary one, and any well-functioning society will have citizens motivated by a healthy degree of shame. The unhealthy side of shame is if the thinking that results from other's disapproval, becomes a crushing and sweeping feeling of a lack of self-worth.

This toxic shame may have its roots in chronic exposure to conditions that cause shame. Conditions, events, or circumstances that cause a question about one's value can cause shame. There are numerous conditions that can trigger shame, triggered by failures or disappointments, and it does not necessarily have to be rooted in childhood trauma. The way that shame feels depends on the person experiencing it, and on the cognition that is involved. For example, if a student fails an entrance exam, the thoughts surrounding the seriousness of the shortcoming, will determine the degree of shame to be felt. In a less serious case, such as making a mistake, forgetting someone's name, or dropping the ball on a project unintentionally, shame may be experienced as a warm sensation, blushing, or a need to divert one's gaze [1].

\section{Manifestations of Shame}

Other manifestations of shame can be shyness and selfconsciousness, especially when a person is fearful of doing something that might cause embarrassment. Some psychologists consider bullying as an externalization of one's unresolved feelings 
of shame and inadequacy. When a person has the feeling that nothing, they do is correct, or meets needs and expectations of others, they are experiencing an inferiority complex. Most psychologists will attest to an inferiority complex as the most severe display of shame. Withdrawal in many ways to avoid any disapproval is a typical coping mechanism with this form of shame. Withdrawal and being disengaged from loved ones and friends, often triggers the very thing the person is trying to avoid, which is disapproval.

This disapproval can intensify the feelings of shame for the person and is problematic if it leads to any form of self-harm. Some positive interventions for lessening or removing shame can be to amend personal standards. By doing so, one can allow for imperfection, not blame themselves when a situation does not merit it and admitting feelings of shame to a trusted friend. Also, talking things through, especially as they pertain to one's shame, can shed new light, and provide a more accurate perspective that reduces or eliminates shameful feelings [1].

\section{Shame and Guilt: Together but Separate}

Shame can be experienced as a desire to avoid a particular circumstance, person, or environment, related to the transgression, or a reminder of it. Often times, shame and guilt are coupled together however there are some notable differences. The most significant difference is that guilt is attached to a particular event. A second component of guilt is behavior that was intentional. Shame, on the other hand, is an emotional experience of an allencompassing failure. Sometimes this emotional experience is because of unintentional actions or not taking any action at all. Some degree of shame is necessary in aiding to conform behavior as appropriate. However, disproportionate or undeserved shame may adversely burden a person's psychological, and by extension, their physical health.

Shame that is unhealthy, or toxic, is about a person's beliefs; specifically, if these beliefs are a threat to one's social position, including fear of devaluation and rejection [2]. There are several universal, classic behavioral responses to shame, to include hiding and social withdrawal. One tragic perpetuation of childhood abuse, if shame has its origins in this regard, is that the experience of devaluation and rejection hinders the person from disclosing what happened. This culminates in a lonely and isolated existence, even in the presence of other people.

Researchers Dodson and Beck (2017) found that shame was a mediator between Post-Traumatic Stress Disorder (PTSD) symptoms and negative anticipations to social networks [3]. If the origins in one's shame-based experiences are related to violence, it may reduce the individual's ability to protect themselves or seek out necessary protection. Also, shamed-based people as a result of interpersonal violence may motivate the person to pursue maladaptive peer groups. One possible explanation for this is because of shame-related beliefs that other social alternatives do not have. Another explanation is that having unhealthy, unwarranted shame may obstruct with self-protection and selfassertion.

Because shame is so painful, people may use alcohol or other substances to assuage the pain. In an endless loop, without a mitigating intervention, these behaviors can place them at risk of victimization. Such victimization can include a repeat of similar family of original relationship patterns and dynamics, risky behaviors, poor self-care that leads to harm, and other painful repetition compulsions. Another major adaptation of unresolved shame is that shame-based people tend to keep secrets. Maintaining a secret vault, sealed off by shame, comes at a price to one's well-being. Secrecy has been found to be interrelated with depression, anxiety, and lower physical health. The disguise of secrecy causes distraction, loneliness, disconnect, and places a barrier to true intimacy with others. Interesting to note, is that what reliably predicts lower well-being is the frequency of mind wandering to (not concealing) secrets [4].

It is pertinent to the discussion of shame and emotions, to specify that shame is a self-conscious emotion. As a self-conscious emotion, shame has more important consequences for secrecy, dissimilar to more basic emotions, like anger and fear for example. This is because basic emotions like anger, fear, or joy, can denote to external targets. Emotions that are of the self-conscious variety, center on the self, therefore, secrets often entail negative selfrelevant information. Therefore, negative self-conscious emotionsstimulated through contemplation on how an event is relevant to self-representations-most likely will continue to fuel shame [4]. In mapping out shame and relevant guilt, it is helpful to note that these emotions reside in the private self. The private self contains one's secrets, in contrast to embarrassment, for example, which refers to the public self.

Shame-related emotions are frequently associated with moral reasoning, and secrets usually contain moral violations [5]. The advantage of linking guilt and shame with different appraisals and phenomenologies is to increase emotional literacy. If addressing it in the context of a treatment plan, knowing what emotion is typically associated with what behavior provides for a targeted intervention. For example, since guilt is usually attendant with negative evaluations of the one's behavior coupled with feeling remorse, addressing the concrete reasons for the guilt would be a good start. If the client was raised in a guilt-induced environment, dishonestly assigned guilt and blame, or other maladaptive coping styles, the client may benefit from taking a close, inventoried look at the behaviors they feel are not moral.

Sometimes conducting a reality-check, asking the client to itemize their guilt-inducing behaviors, can provide a greater awareness and clarity, thereby reducing the guilt. As previously stated, shame is the product of having a negative appraisal of the self and feeling helpless or small [5]. Often, in traumatic experiences, shame results when one feels that they could have been effective or prevented the trauma. Shame runs deep and throughout families where inter-generational problems, addictions, and compulsions have gone unattended.

\section{Shame and Guilt as Distractors}

Recent studies suggest that having to keep a secret hidden in a social interaction should predominantly track how often one encounters conversations related to the secret topic. However, repetitive mind wandering to a secret should track more maladaptive coping efforts [6,7]. Therefore, it follows that shame and guilt will be correlated to mind wandering rather than a coverup. In addressing the aspects of guilt with a client, it is important to bring to the client's awareness, their negative evaluation of the behavior, and subsequent remorse.

This provides a clear starting point for the therapist to prompt the client in discussing their adaptive coping mechanisms with stressors. With regards to features of shame, which is a negative evaluation of the self, and feeling helpless, it would be helpful to prompt the client to discuss their maladaptive coping skills and attitudes. The treatment goal would be to minimize the shamebased repetitive mind wandering to a secret, and with their guilt, to lessen their mind wandering to a secret. Hopefully, this leads the client to more inner resolution, and more adaptive, consciousness, aware, coping responses. 
Considering the origin of the shame-wound is critical as well. If one is the child of abusive and/or addicted parents, their shame could have been an emotional and psychological 'transplant'. Assessing if the shame is a consequence of actions, inactions, or someone else's conflicts, can allow for internal conflict resolution.

\section{Conclusion}

Can we voluntarily control our emotions, including shame? This is an important question, and the answer is that emotions can be controlled. A healthy approach to controlling emotions would include sufficient self-awareness of emotional triggers and practicing of appropriate coping skills. Without a decision to be self-aware, have good impulse control, and regular selfassessment, emotions will run the show. Shame can be triggered when feeling stressed, angry, in-love, or by other emotions that involve our self-worth. Overall, people want to feel valued, with self-worth, and worthy of love and belonging. There are situations that we do not have control over our environment, but we can be good stewards of our thoughts. This gets better with time and diligent practice.

It is advisable to live in, and continually co-create an environment that is supportive, healthy and "other-centered". Shame and guilt thrive in environments that are self-centered, critical, intolerant, or abusive. Removing oneself may spare a lot of ongoing and unnecessary aggravation. Otherwise, the average person will end up influenced and triggered in some way. This can create unnecessary suffering from and can hinder gains made in therapy. Building a reservoir of positive life actions, to increase self-esteem as a buffer for shame, involves a supportive environment, beginning with one's thoughts. Practicing patience is critical to the process of healing shame, reducing the intensity of triggers, and choosing healthy responses when triggered. It can be likened to a person who is recovering from an illness or operation and must take caution not to undo the effects of the treatment, for as long as it takes.
As hard as it may be for the person, without patience, they can do serious damage to themselves. So too, a person who has made strides in lessening a shame trigger must be exceedingly careful in that area, even after they no longer experience significant interference with it. Gaining an increased level of awareness, selfcompassion, more emotional freedom, true understanding and knowledge, combined with the right action, may lessen pain and increase genuine joy. All of these are advisable actions, thought processes and goals, in moving towards a shame reduction trajectory.

\section{References}

1. Ungvarsky J. Shame (social emotion) (2019) Salem Press Encyclopedia.

2. Aakvaag HF, Thoresen S, Strøm IF, Myhre M and Hjemdal OK. Shame predicts revictimization in victims of childhood violence: A prospective study of a general Norwegian population sample (2019) Psychol Trauma: Theory, Res, Prac Policy 11: 43-50. https://doi.org/10.1037/tra0000373

3. Dodson TS and Beck JG. Post-traumatic stress disorder symptoms and attitudes about social support: Does shame matter? (2017) J Anxiety Dis 47: 106-113. https://doi.org/10.1016/i.janxdis.2017.01.005

4. Slepian ML, Kirby JN and Kalokerinos EK. Shame, guilt, and secrets on the mind (2019) Emotion. https://psycnet.apa.org/doi/10.1037/emo0000542

5. Tracy JL and Robins RW. The self in self-conscious emotions: A Cognitive appraisal approach (2007) Tracy JL, Robins W and Tangney JP. (Eds) NY: Guilford Press, New York, pp. 3-20. https://doi.org/10.1037/e633912013-033

6. Slepian ML, Chun JS and Mason MF. The experience of secrecy (2017) J Personality Soc Psychol 113: 1-33.

7. Slepian ML and Moulton-Tetlock E. Confiding secrets and well-being (2018) Soc Psychol Personality Sci 10: 472-484. https://doi.org/10.1177/1948550618765069 